[15] R. S. Varga, Matrix Iterative Analysis. Englewood Cliffs, NJ: PrenticeHall, 1962.

[16] M. Vidyasagar, Nonlinear Systems Analysis. Englewood Cliffs, NJ: Prentice-Hall, 1993

[17] A. J. Viterbi, CDMA: Principles of Spread Spectrum Communication. Reading, MA: Addison-Wesley, 1995.

[18] R. D. Yates, "A framework for uplink power control in cellular radio systems," IEEE J. Select. Areas Commun., vol. 13, pp. 1341-1347, Sept. 1995.

[19] J. Zander, "Performance of optimum transmitter power control in cellular radio systems," IEEE Trans. Veh. Technol., vol. 41, pp. 57-62, Jan. 1992.

[20] — "Distributed cochannel interference control in cellular radio systems," IEEE Trans. Veh. Technol., vol. 41, pp. 305-311, Aug. 1992.

[21] Third Generation Partnership Project (3GPP). RF System Scenarios, Release 5. 3GPP TR 25.942, v5.1.0 (2002-06) [Online]. Available: http://www.3gpp.org

\section{Hierarchical Implicit Deregistration With Forced Registrations in 3G Wireless Networks}

\author{
Yang Xiao, Yuguang Fang, and Yi-Bing Lin
}

\begin{abstract}
Deregistration due to the departures of mobile users from their current visiting registration area may cause significant traffic in the wireless cellular networks. In this paper, we propose a hierarchical implicit deregistration scheme with forced registration in third-generation wireless cellular networks to reduce the remote/international roaming signaling traffic when home-location registers (HLRs), gateway-location registers (GLRs), and the visitor-location registers (VLRs) form a three-level database hierarchy. In this scheme, if a mobile phone arrives and the GLR/VLR is full, a random record is deleted and the reclaimed storage is reassigned to the new arriving mobile phone. When a call arrives and the callee's record is missing in the GLR/VLR, forced registration is executed to restore the GLR/VLR record before the call-setup operation proceeds. An analytic model is proposed to carry out the performance evaluation for the proposed scheme. Our results show that the proposed scheme not only reduces the local deregistration traffic between the GLR and the VLR, but also reduces the remote/international deregistration traffic between the HLR and the GLR, especially when the ratio of the cost of the remote/international traffic between GLR and HLR to the cost of local traffic between the VLR and the GLR is high.
\end{abstract}

Index Terms-Deregistration, gateway location register, location management, third-generation (3G) cellular networks.

\section{INTRODUCTION}

The ANSI-41 [1] and global system for mobile communication (GSM) mobile-application part (MAP) [2] are standards for the mobility management in second-generation $(2 \mathrm{G})$ wireless cellular networks. The ANSI-41 supports advanced mobile-phone service (AMPS), IS-54/IS-136 time-division multiple-access (TDMA) systems (DAMPS), and IS-95 code-division multiple-access (CDMA)

Manuscript received December 25, 2001; revised June 28, 2003 and September 17, 2003. The work of Yuguang Fang was supported in part by the NSF Faculty Early Career Development Award under Grant ANI-0093241.

Y. Xiao is with the Computer Science Department, The University of Memphis, Memphis, TN 38152 USA (e-mail: yangxiao@ieee.org).

Y. Fang is with the Department of Electrical and Computer Engineering, University of Florida, Gainesville, FL 32611 USA (e-mail: fang @ece.ufl.edu).

Y.-B. Lin is with Providence University, Taichung, Taiwan, R.O.C. (e-mail: liny@csie.nctu.edu.tw).

Digital Object Identifier 10.1109/TVT.2003.819817 systems in North America while the MAP is used in GSM 900, DCS1800, and DCS-1900 systems in Europe. Both ANSI-41 and MAP use a two-tier system of home- and visited-location databases. The home-location register (HLR) database is used to store mobile users' personal information in the home system while the visitor-location register (VLR) is used to store mobile users' temporary profile information when a mobile user enters a visited registration area (RA). Registration is the process by which a mobile user informs the network of its current location. Deregistration is the process by which a mobile user informs the old VLR that it has moved out of the old RA. In ANSI-41 [1] and GSM MAP [2], explicit deregistration is used, i.e., whenever a mobile leaves a RA, it will register in the new VLR. A deregistration message will be sent to the old VLR to remove its mobile's profile information. However, in such an explicit deregistration scheme, both registration and deregistration may result in significant network-signaling traffic. In [3], we proposed an implicit deregistration scheme that totally eliminates the deregistration traffic. In this scheme, the record of a mobile is not deleted from the VLR when the mobile leaves that RA. When a mobile arrives at a RA while the VLR is full, a randomly selected record is deleted and the reclaimed storage is then assigned to the new arriving mobile. However, this may cause a problem. When a call to a mobile arrives and the mobile's record is missing from the VLR, the call is lost. To solve this problem, in [4] we improved our implicit-registration scheme as follows. If a call to a mobile arrives and the mobile's record is not in the VLR, a forced registration is executed to restore the VLR record before the call-setup operation proceeds. In this paper, we generalize this scheme to third-generation $(3 \mathrm{G})$ wireless cellular networks by introducing a hierarchically implicit deregistration scheme with forced registration.

With an increasing rate of international travel, the number of roaming users increases. In order to reduce the international (or remote) roaming signaling traffic, the gateway-location register (GLR) within the universal mobile telecommunication system (UMTS) core network is proposed in specification 3GPP 23.119 [5]. The GLR, located in the visited network, is a node between the VLR and/or serving GPRS support node (SGSN) and the HLR. It handles the location management of roaming subscribers in a visited network without involving the HLR in every change of RAs.

In this paper, we present a new location-management scheme, called the hierarchical implicit deregistration scheme, when GLRs are deployed in the $3 \mathrm{G}$ cellular networks. In this scheme, the GLRs, VLRs, and home-location registers (HLRs) form a three-level database hierarchy, where implicit deregistration and forced first/subsequent registration will be used to reduce the signaling traffic consisting of remote/international calls. An analytical model is given to carry out the performance evaluation for the proposed scheme. Although our analytic model is similar to that in [4], the analytical method becomes more complicated when GLRs are deployed.

This paper is organized as follows. In Section II, we briefly discuss the mobility management in the UMTS with the presence of GLRs. Section III describes our hierarchical implicit deregistration scheme with forced first/subsequent registration. In Section IV, we then develop an analytical model to evaluate the performance of our new scheme. Illustrative numerical examples are given in Section V to show the effectiveness of our proposed scheme. Finally, we conclude this paper in Section VI.

\section{MobILITY MANAGEMENT WITH GLRS IN THE UMTS}

In this section, we present the mobility management for $3 \mathrm{G}$ wireless cellular systems (particularly UMTS), in which GLR is normally deployed at the edge of the visited networks. A GLR contains a roamer's 


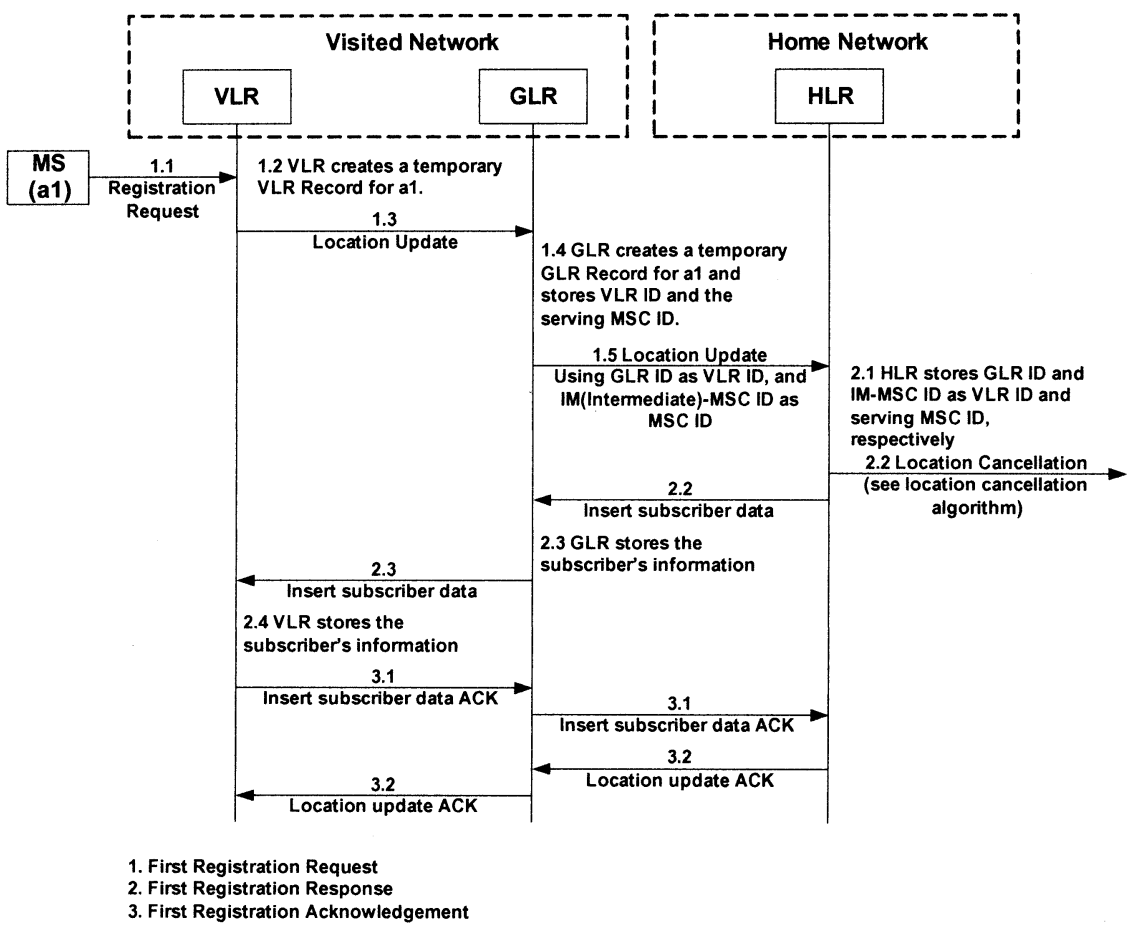

Fig. 1. First registration.

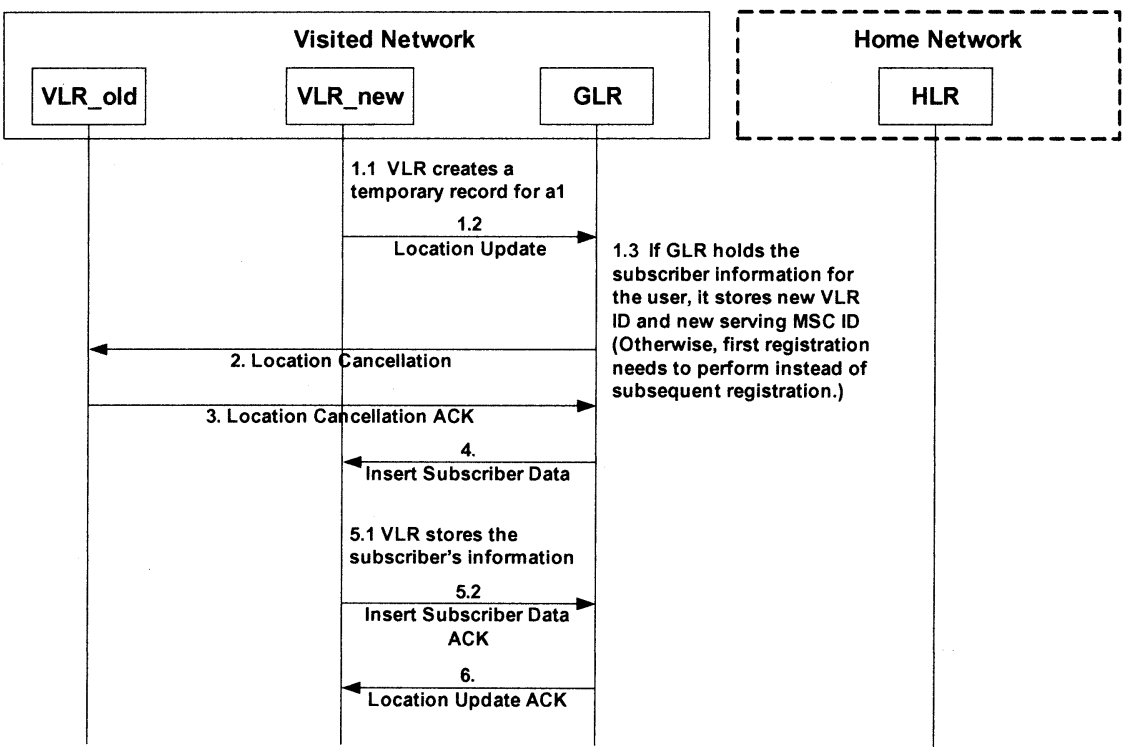

Fig. 2. Subsequent registrations.

subscriber-profile information. At the first location-update procedure under the GLR, the subscriber-profile information is downloaded from the HLR to the GLR. The GLR handles update-location messages from the VLRs as if it were the HLR of the subscribers at the subsequent location updates. This enables a procedure that is invisible from the home network so that the costly internetwork signaling due to location management can be minimized. The GLR keeps the profile information until a cancel-location message is received from the HLR. Location cancellation is the same as the deregistration used in $2 \mathrm{G}$ cellular networks. A GLR can interact with multiple VLRs.

There are five algorithms used in the location management: first registration (shown in Fig. 1), subsequent registration (shown in Fig. 2), location cancellation (deregistration; shown in Fig. 3), call origination, and call termination. The first three algorithms are used for the location update. The last two algorithms are used for call operations. With the location-update procedures, both the HLR and GLR maintain the current location information of every mobile in the visited system under the GLR and guarantee that the mobile user has exactly one VLR record. A first registration is performed when an MS moves to a visited network or an inter-GLR location update is needed, whereas a subsequent registration is performed when an intra-GLR location update is needed. After the first registration, the subsequent registrations are performed between the GLR and the VLRs. The call-origination procedure sets up a call connection initialed by a mobile user using the VLR record. The first registration is handled by the HLR and the VLR via the GLR. For all subsequent registration operations, the HLR is no longer involved and the operations are performed locally between the VLRs and the charging GLR. 


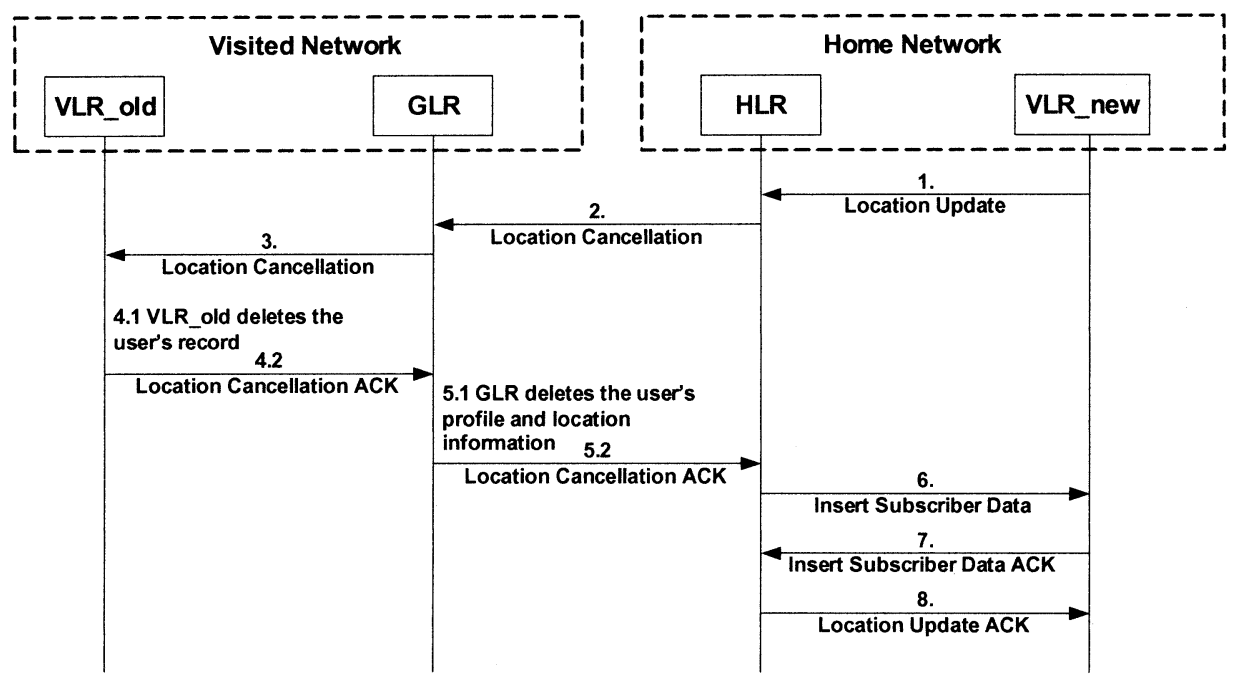

Fig. 3. Location cancellation.

\section{A. Call-Origination Algorithm}

Step 1) The mobile sends the call-origination request to the current MSC.

Step 2) The MSC forwards the request to the VLR.

Step 3) The VLR checks the mobile's profile and forwards the request to the GLR.

Step 4) The GLR checks the mobile's profile and forwards the request to the HLR.

Step 5) The HLR checks the mobile's profile and performs authentication at the authentication center, which may or may not collocate with the HLR. If successful, the HLR grants the call request.

Step 6) The MSC sets up the trunk according to the SS7 call-setup procedure as in $2 \mathrm{G}$ wireless systems.

\section{B. Call-Termination Algorithm}

Step 1) The calling mobile/fixed phone sends a request to its location switch (an MSC if mobile), which forwards the request to the HLR. The HLR forwards the request to the IM-MSC and then to the GLR, based on the location information in the called user's record.

Step 2) The GLR checks the user's profile and forwards the request to the MSC and then to the VLR, based on the location information in the called user's record.

Step 3) The VLR checks the user's record and finds the temporary ID. Paging is performed by the MSC using the temporary ID to find a called mobile user. If the user is found, the routable address information is forwarded to the calling switch. The switch sets up the trunk according to the standard SS7 callsetup procedure, as in the $2 \mathrm{G}$ wireless systems.

\section{HIERARCHICAL IMPLICIT DEREGISTRATION WITH FORCED FIRST/SUBSEQUENT REGISTRATION}

When the implicit registration is used, the record of a mobile is not deleted from the VLR when the mobile leaves that RA. In other words, location cancellation is not executed. If a mobile arrives and the VLR is full, a randomly selected record is deleted and the reclaimed storage is reassigned to the newly arriving mobile. If a call arrives and the called mobile's record is not in the VLR, the forced first/subsequent registrations are executed to restore the VLR record before the call-setup operation starts. To implement the implicit deregistration, we propose the following VLR/GLR algorithms: revised first registration, revised subsequent registration, and revised call origination. Notice that the location-cancellation algorithm is eliminated.

1) Revised First Registration: The following revisions are required in Fig. 1.

- In Step 1.2), if the VLR is not full, Step 1.2 is executed. Otherwise, a randomly chosen record (with equal probability) is deleted and the reclaimed storage is reassigned for $a_{1}$.

- In Step 1.4), if the GLR is not full, Step 1.4 is executed. Otherwise, a randomly chosen record in the GLR (with equal probability) is deleted and the reclaimed storage is reassigned for $a_{1}$ and stores the VLR ID and serving MSC ID.

- In Step 2.2), the location-cancellation procedure will not be initiated.

2) Revised Subsequent Registration: The following revisions are needed in Fig. 2.

- In Step 1.1), if the VLR is not full, it creates a temporary VLR record for $a_{1}$. Otherwise, a randomly chosen record (with equal probability) is deleted and the reclaimed storage is reassigned to $a_{1}$. The VLR sends a location-update message to the GLR.

- In Step 1.2), when the GLR receives a location-update message from the newly visited VLR. If it holds the subscriber information for the user, the GLR stores the new VLR ID and the new serving MSC ID. Otherwise, a randomly chosen record (with equal probability) is deleted and the reclaimed storage is reassigned to $a_{1}$ and stores the VLR ID and the serving MSC ID.

- Steps 2) and 3) are skipped, i.e., the location-cancellation procedure is not initiated.

\section{A. Revised Call Origination}

- In Step 3), if the VLR cannot find the user's record, the VLR forwards the call request to the GLR, along with the revised first registration request.

- In Step 4), if the GLR cannot find the user's record or the GLR receives the first registration request from the VLR, the GLR forwards the call request to the VLR, along with the revised first registration request.

Note that, due to authentication and other reasons, the call-origination process always needs to contact the GLR and the HLR; therefore, if the records in either the GLR or the VLR are missing, the first registration process can be combined with the authentication to recover the record(s). The overhead will not be significant. 
Mobile phone enters LA

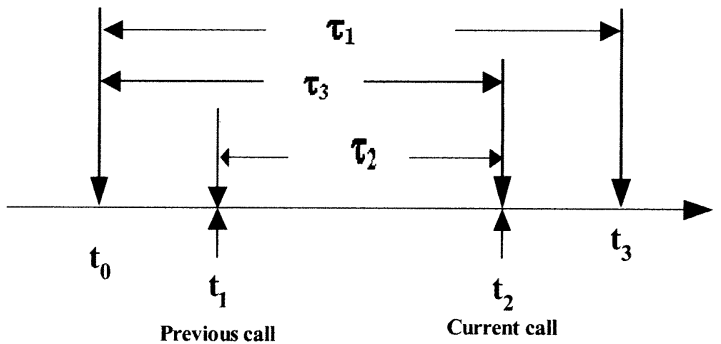

Fig. 4. Timing diagram of mobile $P$ and its calls.

\section{B. Revised Call-Termination Algorithm}

- In Step 2), if the GLR record is missing, the revised first registration is performed by the GLR to recover the GLR record.

- In Step 3), if the VLR record is missing, the revised subsequent registration is performed by the VLR to recover the VLR record.

\section{AnALYTiCAL MODEL}

In [4], the performance of the implicit registration is evaluated in terms of the record-missing probability and the signaling traffic reduction for the $2 \mathrm{G}$ wireless systems. In this paper, we carry out the performance analysis for the proposed scheme for $3 \mathrm{G}$ wireless systems with the presence of the GLR. We observe that, in 3G wireless networks, the signaling traffic between the HLR and GLR is usually remote/international with a high cost, while the signaling traffic between the GLR and VLR is usually local with low cost. In this section, we will study the record-missing probability for both the GLR and VLR, and the gains from the traffic reduction between the HLR and GLR (the international/remote traffic) and between the GLR and VLR (local traffic). To do so, we invoke the following assumptions:

1) Assumption A: The residence time $\tau_{1}$ of a user in a RA follows a general probability distribution with probability density function (pdf) $f_{m}\left(\tau_{1}\right)$, the mean $1 / \eta$, the Laplace transform $f^{*}(s)=\int_{\tau_{1}=0}^{\infty} f\left(\tau_{1}\right) e^{-s \tau_{1}} d \tau_{1}$, and the pdf $F(\cdot)$.

2) Assumption B: The call arrivals to a user form a Poisson process with the call-arrival rate $\lambda$. Let $\tau_{2}$ be the call interarrival time.

Let $N$ be the number of users in a RA, $K$ be the number of VLRs connected to a GLR, $M$ be the size of a VLR, and $L$ be the size of a GLR. From Assumption A, if $N$ is sufficiently large, we can approximate the arrivals of mobiles into a VLR by a Poisson process with rate $N \eta$ [4], [6]. Moreover, the net call arrivals to the mobiles in the VLR form a Poisson process with rate $N \lambda$ [4], [6]. Similarly, we can approximate the arrivals of mobiles into the GLR by a Poisson process with rate $K N \eta$ and the net call arrivals to the mobiles in the GLR by a Poisson process with rate $K N \lambda$. If the VLR record replacement is done randomly with equal probability, the probability that a VLR record is not selected for replacement is

$$
q_{V}=\frac{M-1}{M} .
$$

Similarly, if the GLR record replacement is done randomly with equal probability, the probability that a GLR record is not selected for replacement is

$$
q_{G}=\frac{L-1}{L} .
$$

Fig. 4 shows the timing diagram for a mobile phone $P$ that enters a RA at time $t_{0}$ and leaves the RA at time $t_{3} . \tau_{1}$ is $P$ 's residence time in the RA. Assume that a call to $P$ arrives at time $t_{2}$ where $\max \left(t_{0}, t_{1}\right)<$ $t_{2}<t_{3}$ and the previous call to $P$ arrives at time $t_{1}$. We do not assume that $t_{1}>t_{0}$. Let $\tau_{1}=t_{3}-t_{0}, \tau_{2}=t_{2}-t_{1}, \tau_{3}=t_{2}-t_{0}$, and $\tau=\min \left(\tau_{3}, \tau_{2}\right)$. We notice that $\tau_{2}$ is the call interarrival time both $\tau_{1}$ and $\tau_{2}$ had already defined in assumptions A and B. Since the Poison call arrivals are random observers to $P$ 's residence time, following the residual life theorem [7], $\tau_{3}$ 's density function is

$$
r_{m}\left(\tau_{3}\right)=\eta \int_{t=\tau_{3}}^{\infty} f_{m}(t)=\eta\left[1-F_{m}\left(\tau_{3}\right)\right] .
$$

Let $R_{m}(\cdot)$ be the distribution function of $r_{m}(\cdot)$ and the Laplace transform for $\tau$ be $f^{*}(s)$. We have

$$
\begin{aligned}
f(\tau) & =\int_{t_{2}=\tau}^{\infty} \lambda e^{\lambda \tau_{2}} r_{m}(\tau) d \tau_{2}+\int_{\tau_{3}=\tau}^{\infty} \lambda e^{\lambda \tau} r_{m}\left(\tau_{3}\right) d \tau_{3} \\
& =e^{\lambda \tau}\left\{r_{m}(\tau)+\lambda\left[1-R_{m}\left(\tau_{3}\right)\right]\right\} \\
f^{*}(s) & =\int_{\tau=0}^{\infty} e^{-s \tau} f(\tau) d \tau \\
& =\frac{\lambda s+\lambda^{2}+\eta s\left(1-f_{m}^{*}(\lambda+s)\right)}{(s+\lambda)^{2}} .
\end{aligned}
$$

Let $e_{V}$ denote the event that when a call to a mobile $P$ arrives, the VLR record $r_{V}$ of $P$ does not exist. Let $P\left(e_{V}\right)$ denote the probability that the event $e_{v}$ occurs. $P\left(e_{V}\right)$ is called the record-missing probability for the VLR. Let $e_{G}$ denote the event that when a call to a mobile $P$ arrives ( $P$ is in one of the VLRs in the GLR), the GLR record $r_{G}$ of $P$ does not exist. Let $P\left(e_{G}\right)$ be the probability that the event $e_{G}$ occurs. $P\left(e_{G}\right)$ is called the record-missing probability for the GLR.

Note that $P$ has records in both the GLR and VLR at times $t_{0}, t_{1}$, and $t_{2}$, since the records are potentially restored either by a forced first registration (at $t_{0}$ ) or by a forced subsequent registration (at $t_{0}, t_{1}$ and $t_{2}$ ). Before $t_{0}, t_{1}$ and $t_{2}, P$ 's records in both the GLR and VLR may not exist if the records are replaced due to the hierarchical implicit deregistration. On the other hand, $P$ 's records in either the VLR or GLR may be replaced during the period $\left[\max \left(t_{0}, t_{1}\right), t_{2}\right]$ if either a forced first/subsequent registration or a call setup for another mobile selects $P$ 's record in either the VLR or GLR for replacement during this period. This period is $\tau$. Notice that even though $P$ 's record in the VLR is not replaced, its record in the GLR may still be replaced. On the other hand, if $P$ 's record in the VLR is replaced, its record in the GLR may not be replaced.

When a call to the mobile $P$ arrives at $t_{2}, P$ 's record in the VLR is replaced in the period $\tau$ either by a forced first/subsequent registration or by a call request to another mobile whose VLR record does not exist. The replacement rate due to the forced registration is $N \eta$ and the replacement rate due to call requests is with rate $P\left(e_{V}\right) N \lambda$. The latter statement is obtained as follows: since $N \lambda$ is the rate for call arrivals to mobile phones in the VLR, $P\left(e_{V}\right) N \lambda$ is the rate of call requests for which the corresponding VLR records do not exist. We also notice that $P$ 's record in a VLR is selected for replacement with probability $1-q_{V}$. Let $X_{V}$ be the number of such operations in period $\tau$ that may cause the replacement of a VLR record. Thus, the rate of operations that may cause the replacement of a VLR record is given as

$$
\lambda_{V}^{*}=\left(\eta+P\left(e_{V}\right) \lambda\right) N=\left(\frac{\eta+P\left(e_{V}\right) \lambda}{1-q_{V}}\right)\left(\frac{N}{M}\right) .
$$

Similarly, when a call to a mobile $P$ arrives at $t_{2}$, its record in the GLR is replaced in the period $\tau$, either by a forced first/subsequent registration (with rate $K N \eta$ ) or by a call request to another mobile whose GLR record does not exist (with rate $P\left(e_{G}\right) K N \lambda$ ). Again, the latter statement is obtained as follows: $K N \lambda$ is the rate for call arrivals to mobile phones in the GLR and $P\left(e_{G}\right) K N \lambda$ is the rate of call requests for which the corresponding GLR records do not exist. Moreover, $P$ 's record in the GLR is selected for replacement with probability $1-q_{G}$. 
Let $X_{G}$ be the number of such operations in the period $\tau$, leading to the replacement of a GLR record. Then, the rate of operations that may cause the replacement of a GLR record is

$$
\lambda_{G}^{*}=\left(\eta+P\left(e_{G}\right) \lambda\right) K N=\left(\frac{\eta+P\left(e_{G}\right) \lambda}{1-q_{G}}\right)\left(\frac{K N}{L}\right) .
$$

$X_{V}$ and $X_{G}$ are two Poison random variables with the probability mass functions (pmfs) [7]

$$
\begin{aligned}
& P\left[X_{V}=n\right]=\frac{\left(\lambda_{V}^{*} \tau\right)^{n}}{n !} e^{-\lambda_{V}^{*} \tau} \\
& P\left[X_{G}=n\right]=\frac{\left(\lambda_{G}^{*} \tau\right)^{n}}{n !} e^{-\lambda_{G}^{*} \tau} .
\end{aligned}
$$

Since $f^{*}(s)$ is analytic in the right complex plane by observation, from (4) and power series expansions, the record-missing probabilities $P\left(e_{V}\right)$ and $P\left(e_{G}\right)$ can be expressed as

$$
\begin{aligned}
P\left(e_{V}\right) & =1-\int_{\tau=0}^{\infty}\left\{\sum_{n=0}^{\infty} q_{V}^{n} P\left[X_{V}=n\right]\right\} f(\tau) d \tau \\
& =1-\sum_{n=0}^{\infty}\left\{\frac{\left(-q_{V} \lambda_{V}^{*}\right)^{n}}{n !}\left[\left.\frac{d^{n} f^{*}(s)}{d s^{n}}\right|_{s=\lambda_{V}^{*}}\right]\right\} \\
& =1-f^{*}\left(\lambda_{V}^{*}\left(1-q_{V}\right)\right), \quad \text { by using power series expansion } \\
& =\frac{A_{V}^{2}+\lambda A_{V}-\eta A_{V}\left[1-f_{m}^{*}\left(A_{V}+\lambda\right)\right]}{\left(A_{V}+\lambda\right)^{2}}, \quad \text { by }(4), \\
P\left(e_{G}\right) & =1-\int_{\tau=0}^{\infty}\left\{\sum_{n=0}^{\infty} q_{G}^{n} P\left[X_{G}=n\right]\right\} f(\tau) d \tau \\
& =1-\sum_{n=0}^{\infty}\left\{\frac { ( - q _ { G } \lambda _ { G } ^ { * } ) ^ { n } } { n ! } \left[\left.\frac{d^{n} f^{*}(s)}{d s^{n}}\right|_{s=\lambda_{G}^{*}} ^{*}\left(1-q_{V}\right)\right.\right. \\
& =1-f^{*}\left(\lambda_{G}^{*}\left(1-q_{G}\right)\right), \quad \text { by using power series expansion } \\
& =\frac{A_{G}^{2}+\lambda A_{G}-\eta A_{G}\left[1-f_{m}^{*}\left(A_{G}+\lambda\right)\right]}{\left(A_{G}+\lambda\right)^{2}} \text { by (4), }
\end{aligned}
$$

Both $P\left(e_{V}\right)$ [with (6) and (10)] and $P\left(e_{G}\right)$ [with (7) and (11)] can be computed by the following iterative algorithms, as we did in [4]. Let the pair $\left(P(e), \lambda^{*}\right)$ stand for either the pair $\left(P\left(e_{V}\right), \lambda_{V}^{*}\right)$ or the pair $\left(P\left(e_{G}\right), \lambda_{G}^{*}\right)$.

Step 1) Select the initial value for $P(e)$.

Step 2) Compute $\lambda^{*}$ based on (6) or (7).

Step 3) Let $P(e)$ old $\leftarrow P(e)$.

Step 4) Compute $P(e)$ based on (10) or (11).

Step 5) Let $\delta$ be a predefined small value. If $\left|P(e)-P(e)_{\text {old }}\right|<$ $\delta|P(e)|$, then exit. Otherwise, $P(e)$ old $\leftarrow P(e)$ and go to Step 2.

The above iterative algorithm has been extensively studied and validated by many experiments [6]. Using a similar argument in [4], we can show that the above algorithm converges to the unique solution $P\left(e_{V}\right)$ or $P\left(e_{G}\right)$ and that the convergence is exponentially fast.

For a VLR, let $\mu_{V 1}$ be the saved deregistration traffic between the GLR and VLR in the hierarchically implicit deregistration scheme and $\mu_{V 2}$ be the extra traffic created between the GLR and VLR due to the forced first/subsequent registrations. Then, we have $\mu_{V 1}=N \eta$ and $\mu_{V 2}=P\left(e_{V}\right) N \lambda$. Similarly, for a GLR, let $\mu_{G 1}$ be the saved deregistration traffic between the HLR and GLR in the hierarchically implicit deregistration scheme and $\mu_{G 2}$ be the extra traffic created between the HLR and GLR due to the forced registration. Similarly, we have $\mu_{G 1}=K N \eta$ and $\mu_{G 2}=P\left(e_{G}\right) K N \lambda$. Let $\alpha_{V}$ be the ratio of a deregistration cost to a registration cost for the VLR and $\alpha_{G}$ be the ratio of a deregistration cost to a registration cost for the GLR. In a typical mobile phone network, we have $1<\alpha_{V}<2$ and $1<\alpha_{G}<2$ [4]. Let $\sigma$ be the traffic cost ratio of the remote/international traffic cost between the GLR and HLR versus the local traffic between the VLR and GLR. Normally, we expect that $\sigma \gg 1$. The saved remote traffic $\gamma_{\text {remote }}$, the saved local traffic $\gamma_{\text {local }}$, and the saved total traffic $\gamma$ in the hierarchically implicit deregistration scheme for a GLR with $K$ associated VLRs are given as

$$
\begin{aligned}
\gamma_{\text {remote }} & =\sigma\left[\mu_{G 1}-\alpha_{G} \mu_{G 2}\right]=K N \sigma\left[\eta-P\left(e_{G}\right) \alpha_{G} \lambda\right] \\
\gamma_{\text {local }} & =K\left[\mu_{V 1}-\alpha_{V} \mu_{v 2}\right]=K N\left[\eta-P\left(e_{V}\right) \alpha_{V} \lambda\right] \\
\gamma & =\gamma_{\text {remote }}+\gamma_{\text {local }} \\
& =K N\left[(\sigma+1) \eta-\left(P\left(e_{G}\right) \alpha_{G} \sigma+P\left(e_{V}\right) \alpha_{V}\right) \lambda\right]
\end{aligned}
$$

\section{NUMERICAL RESULTS}

In this section, we carry out the performance analysis for our new scheme. We further assume that the RA residence times have a Gamma density function with mean $1 / \eta$ and variance $\nu$ [4]. The Laplace transform for the Gamma RA residence time distribution is

$$
f_{m}^{*}(s)=\left(\frac{1}{1+\eta v s}\right)^{1 /\left(\eta^{2} v\right)} .
$$

Therefore, (10) and (11) can be rewritten as shown in (16) and (17) at the bottom of the page.

Based on (6) and (16), we can calculate $P\left(e_{V}\right)$ using the iterative algorithm introduced in the previous section. Similarly, we can calculate $P\left(e_{G}\right)$ based on (7) and (17). Then, we can calculate $\gamma_{\text {remote }}, \gamma_{\text {local }}$, and $\gamma$ using the values of $P\left(e_{V}\right)$ and $P\left(e_{G}\right)$ based on (12)-(14). Based on $\gamma_{\text {remote }}, \gamma_{\text {local }}$, and $\gamma$, we investigate the performance of the hierarchically implicit deregistration with forced first/subsequent registration as follows.

Fig. 5 plots the probabilities $P\left(e_{V}\right)$ and $P\left(e_{G}\right)$ versus $\lambda / \eta$ (the expected number of calls to a mobile phone when a mobile is in a RA). Here, we assume that $\nu=0.1 / \eta^{2}, 1 / \eta^{2}, 10 / \eta^{2}$ in the hierarchically implicit deregistration scheme for a GLR. The figure indicates that both probabilities decrease as $\lambda / \eta$ increases. That is, if the mobile's mobility is high or if the call-arrival rate is low, it is more likely that when a call arrives, the corresponding VLR and GLR records have been replaced. As $\nu$ increases, the probabilities increase, but the difference becomes less as $\lambda / \eta$ increases.

Fig. 6 plots the saved total traffic $\gamma$ versus $\lambda / \eta$ (the expected number of calls to a mobile when a mobile is in a RA). Here, we assume that $M=10000, N=3000, L=20000, \alpha_{G}=1, \alpha_{V}=1, \sigma=$ $1.5,3.0,5.0$, and $\nu=0.1 / \eta^{2}, 1 / \eta^{2}, 10 / \eta^{2}$ in the hierarchically implicit deregistration scheme for a GLR with two associated VLRs. The figure indicates that by exercising the hierarchically implicit deregistration, the portion of deregistration traffic can be significantly reduced,

$$
\begin{aligned}
& P\left(e_{V}\right)=\frac{A_{V}^{2}+\lambda A_{V}-\eta A_{V}\left[1-\left(1+\eta v A_{V}+\eta v \lambda\right)^{-1 /\left(\eta^{2} v\right)}\right]}{\left(A_{V}+\lambda\right)^{2}} \text { where } A_{V}=\lambda_{V}^{*}\left(1-q_{V}\right) \\
& P\left(e_{G}\right)=\frac{A_{G}^{2}+\lambda A_{G}-\eta A_{G}\left[1-\left(1+\eta v A_{G}+\eta v \lambda\right)^{-1 /\left(\eta^{2} v\right)}\right]}{\left(A_{G}+\lambda\right)^{2}} \text { where } A_{G}=\lambda_{G}^{*}\left(1-q_{G}\right) .
\end{aligned}
$$




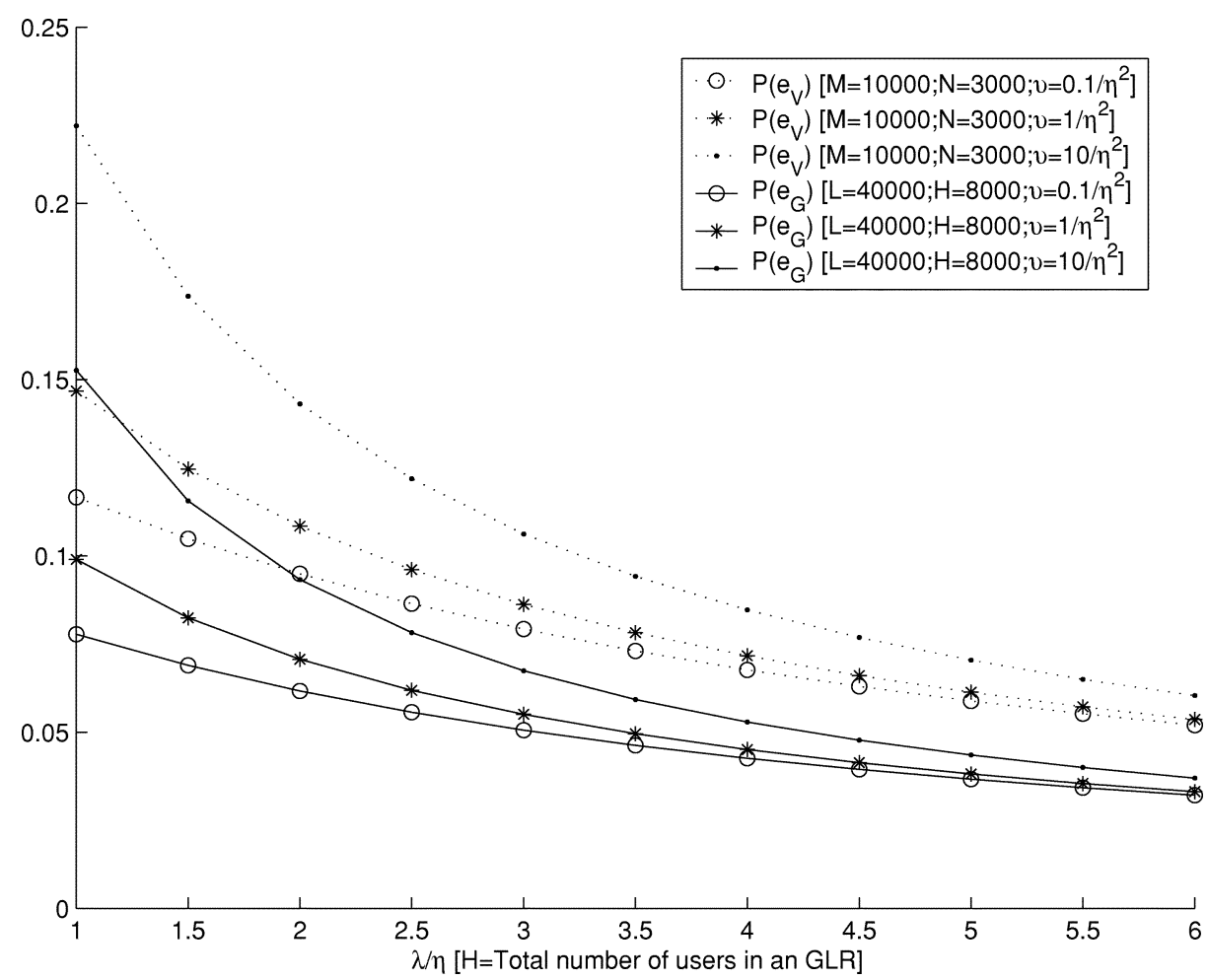

Fig. 5. Probabilities $P\left(e_{V}\right)$ and $P\left(e_{G}\right)$ versus $\lambda / \eta$.

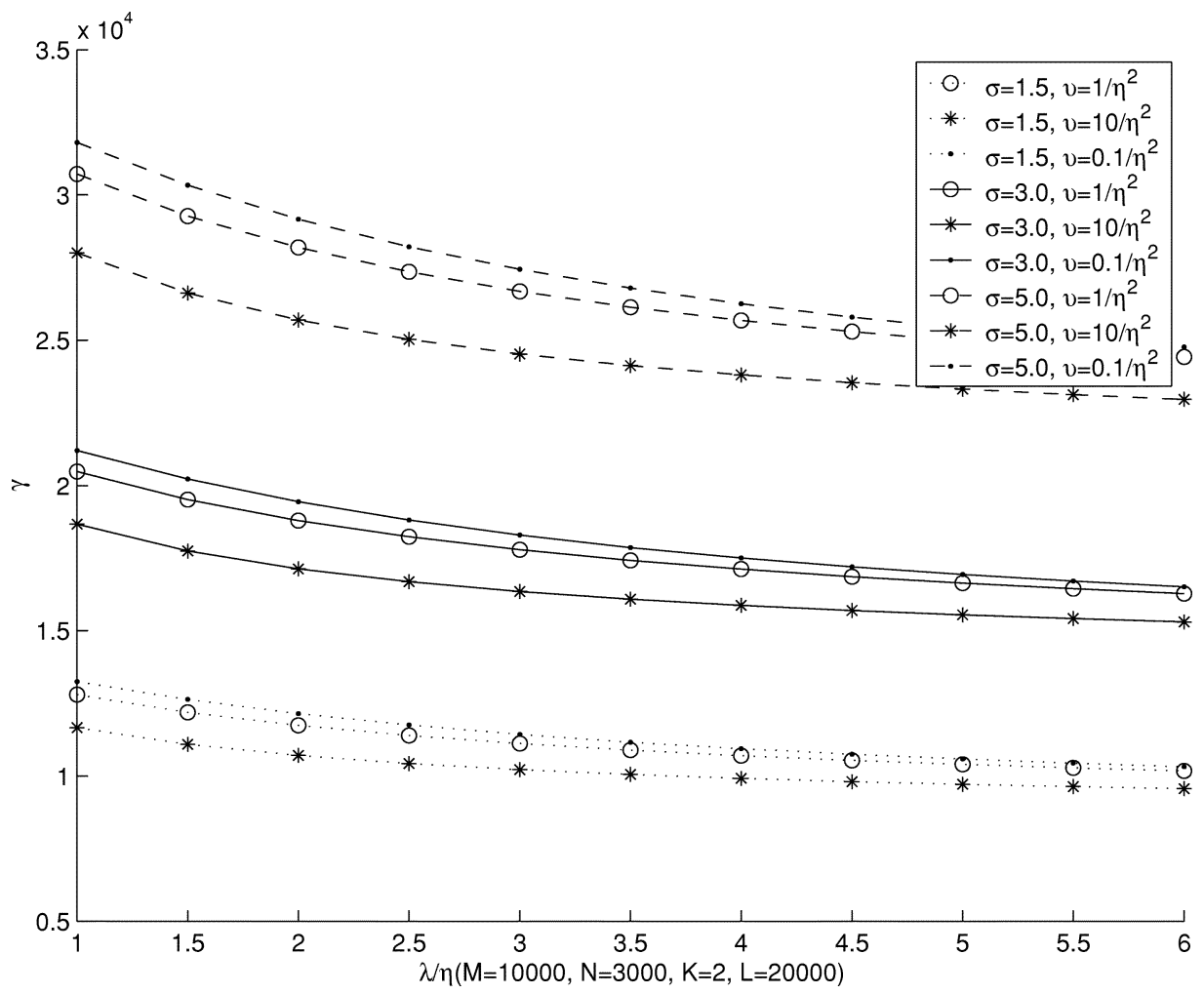

Fig. 6. Effects of $\sigma, \nu$ and $\lambda / \eta$ on $\gamma$.

especially when $\sigma$ is high. We expect $\sigma$ is higher when the traffic between the GLR and HLR is international traffic. The figure also shows that the saved total traffic decrease as $\lambda / \eta$ increases. That is, if the mobile's mobility is high or the call-arrival rate is low, it is more likely that when a call arrives, the corresponding VLR and GLR records have been replaced. Furthermore, the figure demonstrates how the variance $\nu$ of the Gamma cell residence time distribution affects the system performance with a fixed mean $1 / \eta$ : the saved total traffic in the hierarchical implicit deregistration decreases as $\nu$ increases.

Fig. 7 plots the saved total traffic $\gamma$ versus $\lambda / \eta$ with $\sigma=3.0, L=$ $2 * M, \nu=1 / \eta^{2}, \alpha_{G}=1, \alpha_{V}=1, M=10000,5000,1000$, and $N=0.1 M, 0.3 M, 0.9 M$ in the hierarchical implicit deregistration 


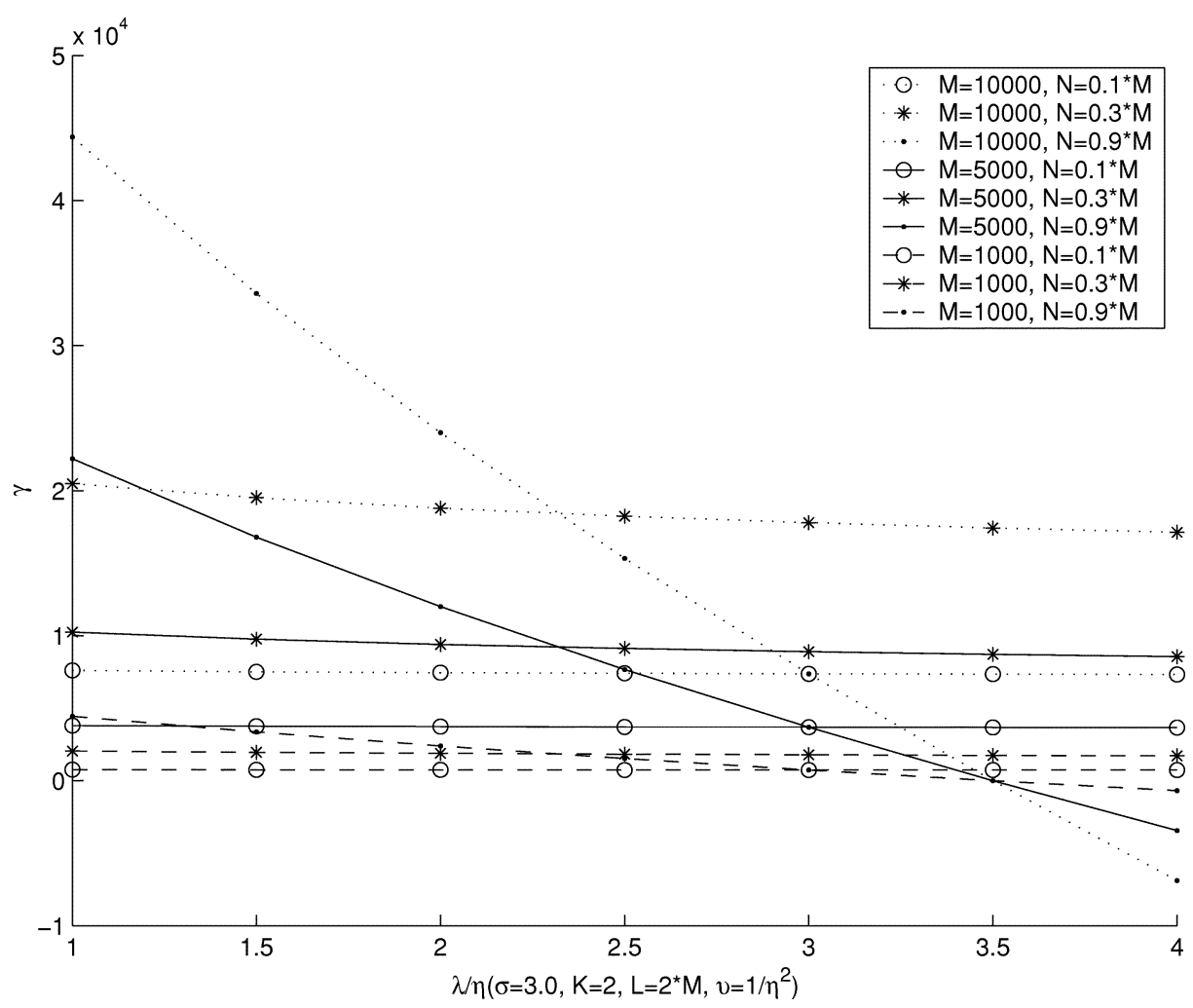

Fig. 7. Effects of $M, N$, and $\lambda / \eta$ on $\gamma$.

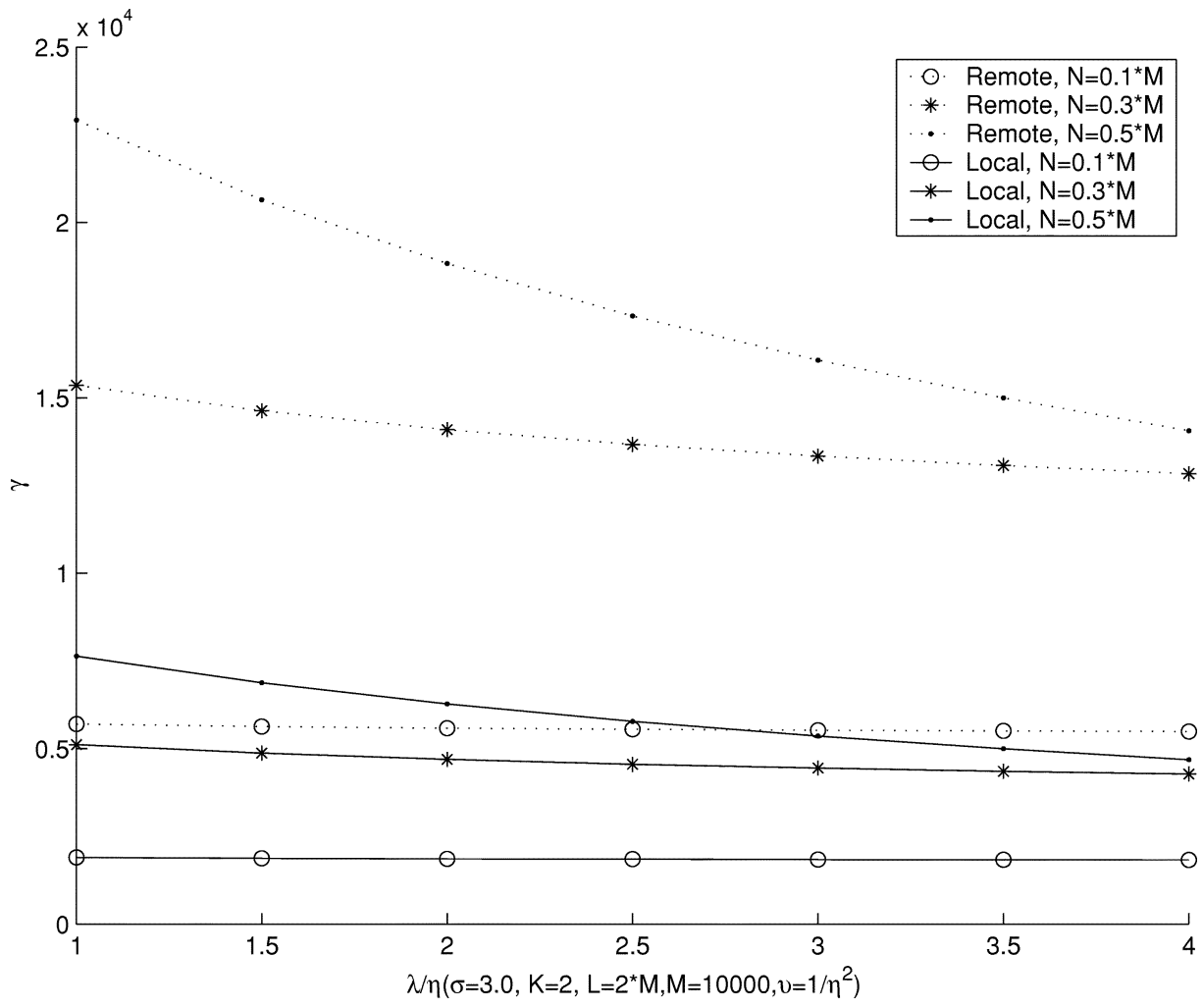

Fig. 8. Saved remote traffic versus saved local traffic.

for a GLR with two associated VLRs. The figure shows that by exercising the hierarchically implicit deregistration, the portion of deregistration traffic can be significantly reduced, especially when $M$ (the size of the VLR) is large. The saved traffic increases when $M$ increases.
The figure also shows that the saved total traffic increases significantly when $N / M$ increases. Furthermore, the figure demonstrate that when $M$ is large $(M \geq 10000)$ and $N / M$ is large $(N / M \geq 0.3)$, the saved total traffic decreases as $\lambda / \eta$ increases. That is, when both $M$ and 
$N / M$ are large, if the mobile phone's mobility is high or the call-arrival rate is low, in which case, it is more likely that when a call arrives, the corresponding VLR and GLR records will be replaced. On the other hand, when $M$ is small $(M<10000)$ and $N / M$ are small $(N / M<0.3)$, the saved total traffic is insensitive as $\lambda / \eta$ increases. However, when $N$ is close to $M$, i.e., $N=0.9 M$, and $\lambda / \eta$ is large, close to 4.0, the performance of the proposed scheme becomes worse than the original scheme. In other words, when the sizes of databases are small and call-to-mobility increases, the proposed scheme will become worse. We recommend that the size of the databases should be large enough (such as twice of the number of users in the area) so that the proposed scheme will perform better.

Fig. 8 plots the saved remote traffic $\gamma_{\text {remote }}$ and the saved local traffic $\gamma_{\text {local }}$ versus $\lambda / \eta$ with $\sigma=3.0, L=2 * M, \nu=1 / \eta^{2}, \alpha_{G}=1, \alpha_{V}=$ $1, M=10000$, and $N=0.1 M, 0.3 M, 0.5 M$ in the hierarchically implicit deregistration for a GLR with two associated VLRs. The figure indicates that by exercising the hierarchically implicit deregistration, the portion of the saved remote traffic can be significantly larger than the saved local traffic, especially when $N / M$ is large. Both the saved remote traffic and the saved local traffic increase when $N / M$ increases.

\section{CONCLUSION}

This paper proposes a hierarchically implicit deregistration scheme to reduce the signaling traffic in $3 \mathrm{G}$ wireless cellular systems. An analytical model is developed to evaluate the performance of the proposed scheme. The saved remote traffic, the saved local traffic, and the total saved traffic are used as the output performance measures. The study indicates that by exercising our hierarchically implicit deregistration, the portion of deregistration traffic can be significantly reduced, especially when the traffic cost ratio of the remote/international traffic cost between the GLR and HLR versus local traffic between the VLR and the GLR is high. Moreover, by exercising the hierarchically implicit deregistration, the portion of the saved remote traffic can be significantly larger than the saved local traffic. The portion of deregistration traffic can be significantly reduced, especially when the size of the VLR is large. The saved traffic increases when the size of the VLR increases. The total saved traffic increases dramatically when the ratio of the number of mobiles to the size of the VLR databases increases. The results can be useful in mobility database dimensioning and quality-of-service provisioning in $3 \mathrm{G}$ wireless network designs.

\section{REFERENCES}

[1] ETSI/TC, “Cellular Intersystem Operations (Rev. C),” Tech. Rep. IS-41, 1995.

[2] ETSI/TC, "Mobile Application Part (MAP) Specification, Version 4.8.0," Tech. Rep., Recommendation GSM 09.02, 1994. ETSI/TC.

[3] Y.-B. Lin and A. Noerpel, "Implicit deregistration in a PCS network," IEEE Trans. Veh. Technol., vol. 43, pp. 1006-1010, July 1994.

[4] A.-C. Pang, Y.-B. Lin, and Y. Fang, "Implicit deregistration with forced registration fo PCS mobility management," Wireless Networks, vol. 7, no. 1, pp. 99-104, 2001.

[5] Gateway Location Register (GLR) - Stage2, Mar. 2000.

[6] Y.-B. Lin, "Modeling techniques for large-scale PCS networks," IEEE Commun. Mag., pp. 102-107, Feb. 1997.

[7] L. Kleinrock, Queueing Systems: Volume I- Theory. New York: Wiley, 1976. 